\title{
Side wall effects on ship model testing in a towing tank
}

\author{
Zhi-Ming Yuan ${ }^{\mathrm{a}}$, Xinshu Zhang ${ }^{\mathrm{b}}$, Chun-Yan $\mathrm{Ji}^{\mathrm{c}^{*}}$, Laibing Jia ${ }^{\mathrm{d}}$, Huaming Wang ${ }^{\mathrm{e}}$, Atilla Incecik ${ }^{\mathrm{a}}$ \\ ${ }^{\mathrm{a}}$ Department of Naval Architecture, Ocean and Marine Engineering, University of Strathclyde, UK \\ ${ }^{\mathrm{b} S}$ School of Naval Architecture, Ocean and Civil Engineering, Shanghai Jiao Tong University, China \\ 'School of Naval Architecture and Ocean Engineering, Jiangsu University of Science and Technology, China \\ ${ }^{\mathrm{d} S}$ Shool of Marine Science and Technology, Northwestern Polytechnial University, China \\ eSchool of Naval Architecture and Mechanical-electrical Engineering, Zhejiang Ocean University, China
}

\begin{abstract}
Due to the existence of the side walls in a towing tank, the measured hydrodynamic forces would present some discrepancies compared to the open sea results. This phenomenon is referred to as the side wall effect. The objective of the present study is to investigate the side wall effects on ship model testing in a towing tank. The method used in the present study involves a 3D panel method based on the Rankine type Green function. Both the steady and unsteady problems were investigated numerically. The numerical results were validated against ship model test results. After the validations, a large scale computations were performed to investigate the parameters which could determine the side wall effects. Two diagrams of side wall effects (one in calm water and the other one in waves), were obtained which showed whether the side wall effect was less than the permissible error to be included in the measured values.
\end{abstract}

Keywords: Side wall effects; Rankine source method; Ship model testing; Shallow water; Forward speed.

\section{Introduction}

A ship model towing tank always has a limited width. Due to the existence of the side walls of the towing tank, the measured hydrodynamic forces would present some discrepancies compared to the open water results. This phenomenon is referred to as the side wall effect. There are many factors which determine the side wall effects. These factors include ship geometry, width $(w)$ and depth $(h)$ of the tank and forward speed of the ship model. In seakeeping tests, the oscillating frequency is another critical parameter which must be taken into consideration. For a certain combination of the above parameters, the measurements from the tests could differ significantly from the open sea results. The object of the present work is to find the relation between the side wall effects and the parameters which determine the side wall effects. According to the purpose of the ship model test in a towing tank, the side wall may affect the measurements in wave-making problems (in calm water) and seakeeping problems (in waves).

The side wall effects on model testing in calm water is not very obvious and therefore it is usually neglected in ship model tests. In calm water, the side wall effects can be simply estimated from Kelvin wave pattern, as shown in Fig. 1. The waves produced in the bow are reflected by the side walls, and these reflected waves will strike the ship if the tank width $(w)$ is very small. The minimum distance $w_{m}$ can be estimated as

$$
w_{m}=L \tan \theta \approx 0.36 L
$$

where $L$ is the length of the ship model. It indicates that if the width of the tank is larger than $0.36 L$, the side wall effects can be neglected in ship model tests in calm water with infinite water depth. It should be pointed out that this minimum distance $w_{m}$ will be modified by the near field local waves produced by a 3D ship. Therefore, $\mathrm{w}_{m}$ is slightly larger than the estimated value from Eq. (1). However, it will not overturn the conclusion that $w_{m}$ is much smaller than ship length $L$. In practice, the width of most of the towing tanks is larger than $w_{m}$. Therefore, the side wall effects are neglected in wave-making problems in deep water, and the studies on side wall effects on calm water model tests are very rare. But ships advancing in a channel has been widely studied (Beck et al., 1975; Mei and Choi, 1987; Norrbin, 1974; Tuck, 1978). Theoretically, these two problems are very similar. The difference is that

*Corresponding author at: School of Naval Architecture and Ocean Engineering, Jiangsu University of Science and Technology No. 2 Mengxi Road, Zhenjiang, China, 212003.

E-mail address: jichunyanjkd@163.com 
in a towing tank, the ship model is usually fixed in the centre line of the tank (as shown in Fig. 1). There is no force (or moment) components in $y$ direction. Whilst for a ship manoeuvring in a canal or channel, it is very difficult to guarantee the ship is always advancing along the centre line of the canal. Therefore, there is a lateral force, as well as a yaw moment acting on the ship (Yuan and Incecik, 2016). More importantly, the side wall effects are often accompanied by shallow water effects. In shallow water, the water depth Froude number $F_{h}\left(F_{h}=u / \sqrt{g h}\right.$, where $u$ is the forward speed and $g$ is the gravity acceleration) becomes a critical parameter which determines the feature of the wave-making resistance. It was found that in a shallow channel, the wave-making resistance experienced a sudden drop at the critical speed (Kirsch, 1966; Newman and Poole, 1962). Doctors (2015) proposed a formula to estimate this drop and found that the wave-making resistance was less for greater values of the tank width or the water depth.

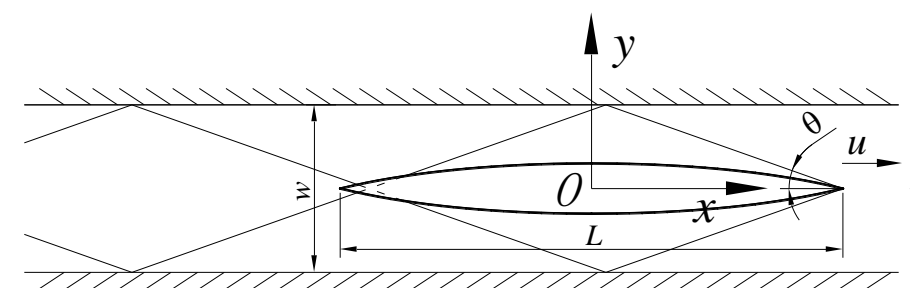

Fig. 1. A sample ship advancing in a towing tank, where $w$ is width of the tank, $L$ is the length of the ship, $u$ is the speed of the model and $\theta$ is the semi-wedge angle of the waves produced by the ship. In calm water, the semi-wedge angle $\theta=\sin ^{-1}(1 / 3) \approx$ $19.47^{\circ}$.

The side wall effects on ship model testing in waves are more complicated than those in calm water due to the factor of oscillating frequency $(\omega)$. In order to investigate the side wall effects on ship model testing in waves, another critical parameter $\tau\left(\tau=\omega_{e} u / g, \omega_{e}\right.$ is the encounter frequency) must be introduced. Due to the oscillating and translating properties, there are three individual wave systems as the parameter $\tau<0.25$ (Becker, 1958; Noblesse and Hendrix, 1992; Yuan et al., 2015b). Correspondingly, the semi-wedge angles are not constant anymore, which is different from Kelvin waves. They are determined by parameter $\tau$. Besides, the semi-wedges angle of the waves produced by an oscillating source are generally larger than Kelvin wedge. Therefore, the side wall effects have to be taken into consideration during the model test in waves in the towing tank. Kashiwagi and Ohkusu $(1989,1991)$ used the asymptotic wave contour to estimate the side-wall effect. They also extended Newman's (Newman, 1978) unified slender-ship theory and developed a new method to calculate the side-wall effects numerically. The critical line obtained numerically was presented and compared to the results estimated from asymptotic wave contour. A diagram which shows whether side wall effects are expected was presented in Kashiwagi's study (Kashiwagi and Ohkusu, 1991). Similar studies were also carried out by Hosoda $(1976 ; 1979)$. These studies are based on slender ship theory. Iwashita (2001) used a 3D Rankine panel method to investigate the unsteady waves in low frequency range. There are many advantages of using Rankine source panel method. But a radiation condition on control surface is required, especially when the parameter $\tau<0.25$.

In this paper, a so-called Sommerfeld radiation condition with forward speed correction is imposed on the control surface to ensure that the waves can propagate to the far field without reflection. This radiation condition is included in our in-house developed program MHydro, which is well validated by experiments (Yuan et al., 2015a; Yuan et al., 2014a; Yuan et al., 2014b; Yuan et al., 2015c).

\section{Mathematical formulation}

\subsection{Boundary value problem (BVP) of steady flow}

When a ship advances at constant speed in calm water, it will generate steady waves and induce the socalled wave-making resistance. It is assumed that the fluid is incompressible and inviscid and the flow is irrotational. The velocity potential can be expressed as

$$
\varphi_{s}=u x+\sum_{j=1}^{\infty} \varepsilon^{j} \varphi_{s j}
$$


where $\varepsilon(\varepsilon<<1)$ is a perturbation parameter which can be defined by $B / L . B$ and $L$ are the breadth and length of the ship. $\varphi_{s}$ satisfies the Laplace equation

$$
\nabla^{2} \varphi_{s}=0 \text { in the fluid domain }
$$

It has been concluded by Tarafder (Tarafder, 2007) that the $2^{\text {nd }}$ order solution could provide a fairly good results. Therefore, only the first two terms of the Taylor series are retained in the present study. The $1^{\text {st }}$ and $2^{\text {nd }}$ order approximations of body surface boundary condition can be expressed as

$$
\begin{aligned}
& \frac{\partial \varphi_{s 1}}{\partial n}=u \cdot n_{1}, \text { on the mean wetted body surface } \\
& \frac{\partial \varphi_{s 2}}{\partial n}=0, \text { on the mean wetted body surface }
\end{aligned}
$$

where $\mathbf{n}=\left(n_{1}, n_{2}, n_{3}\right)$ is the unit normal vector inward on the wetted body surface of the ship. Following Newman (1976), the nonlinear dynamic free-surface condition on the disturbed free surface can be expressed as

$$
u \frac{\partial \varphi_{s}}{\partial x}+\frac{1}{2}\left(\nabla \varphi_{s} \cdot \nabla \varphi_{s}\right)+g \zeta_{s}=0, \text { on } z=\zeta_{s}
$$

The kinematic free-surface condition on the disturbed free surface is

$$
u \frac{\partial \zeta_{s}}{\partial x}-\frac{\partial \varphi_{s}}{\partial z}+\frac{\partial \varphi_{s}}{\partial y} \frac{\partial \zeta_{s}}{\partial y}+\frac{\partial \varphi_{s}}{\partial x} \frac{\partial \zeta_{s}}{\partial x}=0, \text { on } z=\zeta_{s}
$$

Where $\zeta_{s}$ represents instant free surface. The above nonlinear boundary condition can be linearized by using perturbation method. Combining Eq. (6) and (7) and expanding the steady potential in a Taylor series about the mean free surface at $z=0$, the following $1^{\text {st }}$ and $2^{\text {nd }}$ order free surface boundary conditions can be obtained (Maruo, 1966)

$$
\begin{aligned}
& u^{2} \frac{\partial^{2} \varphi_{s 1}}{\partial x^{2}}+g \frac{\partial \varphi_{s 1}}{\partial z}=0, \text { on undisturbed free surface } z=0 \\
& u^{2} \frac{\partial^{2} \varphi_{s 2}}{\partial x^{2}}+g \frac{\partial \varphi_{s 2}}{\partial z}=R_{2}, \text { on undisturbed free surface } z=0
\end{aligned}
$$

where

$$
R_{2}=-\frac{1}{u} \frac{\partial}{\partial x}\left(\nabla \varphi_{s 1} \cdot \nabla \varphi_{s 1}\right)-\zeta_{s 1} \frac{\partial}{\partial z}\left(\frac{\partial^{2} \varphi_{s 1}}{\partial x^{2}}+\frac{g}{u^{2}} \frac{\partial \varphi_{s 1}}{\partial z}\right)
$$

Eq. (10) contains unknown $\zeta_{s l}$ which can be obtained by solving the boundary value problem of the $1^{\text {st }}$ order potential, and it can be expressed as

$$
\zeta_{s 1}=\frac{u}{g} \frac{\partial \varphi_{s 1}}{\partial x}
$$

The boundary condition on the sea bottom and side walls can be expressed as

$$
\frac{\partial \varphi_{s j}}{\partial n}=0, \quad j=1,2 \text {, on } z=-h \text { and side walls }
$$

Here $j=1$ represents $1^{\text {st }}$ order potential and $j=2$ represents $2^{\text {nd }}$ order potential. The radiation condition is satisfied by using second-order upwind differential scheme.

The $1^{\text {st }}$ order potential $\varphi_{s I}$ can be obtained by solving BVP from Eq. (3), (4), (8) and (12). Substituting $\varphi_{s l}$ into Eq. (10), the $2^{\text {nd }}$ order potential $\varphi_{s 2}$ can be obtained by solving BVP from Eq. (3), (5), (9) and (12). The $2^{\text {nd }}$ order contribution to the wave elevation then can be obtained by Taylor's series expansion

$$
\zeta_{s 2}=\frac{u}{g}\left(\frac{\partial \varphi_{s 2}}{\partial x}+\zeta_{s 1} \frac{\partial^{2} \varphi_{s 1}}{\partial x \partial z}-\frac{1}{2 u} \nabla \varphi_{s 1} \cdot \nabla \varphi_{s 1}\right)
$$


The $1^{\text {st }}$ order wave elevation can be written as

$$
\zeta_{s}=\zeta_{s 1}(14)
$$

The $2^{\text {nd }}$ order wave elevation can be written as

$$
\zeta_{s}=\zeta_{s 1}+\zeta_{s 2}
$$

Once the unknown potential $\varphi_{s 1}$ and $\varphi_{s 2}$ are solved, the steady pressure can be obtained from Bernoulli's equation:

$$
\begin{gathered}
p_{s 1}=\rho\left(u \frac{\partial \varphi_{s 1}}{\partial x}-\frac{1}{2} \nabla \varphi_{s 1} \cdot \nabla \varphi_{s 1}\right) \\
p_{s 2}=\rho\left(u \frac{\partial \varphi_{s 2}}{\partial x}-\frac{1}{2} \nabla \varphi_{s 2} \cdot \nabla \varphi_{s 2}-\nabla \varphi_{s 1} \cdot \nabla \varphi_{s 2}\right)
\end{gathered}
$$

The $1^{\text {st }}$ and $2^{\text {nd }}$ order contributions to the steady hydrodynamic forces are obtained by the pressure integral on the wetted body surface as follows:

$$
F_{i}^{s_{j}}=\iint_{S_{b}} p_{s_{j}} n_{i} d S, i=1,2, \ldots, 6 ; j=1,2
$$

The wave making resistance coefficient can be defined as

$$
C_{w j}=-\frac{F_{1}^{s_{j}}}{\frac{1}{2} \rho u^{2} A_{s}}, j=1,2
$$

The mean sinkage $S$ and trim $T$ can be written as

$$
\begin{aligned}
& S_{j}=-\frac{F_{3}^{s_{j}}}{\rho g A_{w}}, \quad j=1,2 \\
& T_{j}=-\frac{F_{5}^{s_{j}}}{\rho g I_{w}}, \quad j=1,2
\end{aligned}
$$

where $A_{s}$ is the area of wetted body surface, $A_{w}$ is the water plane area and $I_{w}$ is second moment of the water plane about the $y$-axis. The $1^{\text {st }}$ order wave making resistance coefficient, mean sinkage and trim can be expressed as

$$
C_{w}=C_{w 1}, \quad S=S_{1}, \quad T=T_{1}
$$

The $2^{\text {nd }}$ order wave making resistance coefficient, mean sinkage and trim can be expressed as

$$
C_{w}=\sum_{j=1}^{2} C_{w j}, \quad S=\sum_{j=1}^{2} S_{j}, \quad T=\sum_{j=1}^{2} T_{j}
$$

\subsection{Boundary value problem of unsteady flow}

It is assumed that the surrounding fluid is inviscid and incompressible, and that the motion is irrotational, the total velocity potential exists which satisfies the Laplace equation in the whole fluid domain. Let $t$ denote time and $\mathbf{x}=(x, y, z)$ the position vector. A complex velocity potential provides a description of the unsteady flow as

$$
\psi(\mathbf{x}, t)=\operatorname{Re} \sum_{j=0}^{7} \eta_{j} \varphi_{j}(x, y \cdot z) e^{-i \omega_{e} t}
$$

where $\varphi_{j}(j=1,2, \ldots, 6)$ are the spatial radiation potential in six degrees of freedom corresponding to the oscillations of the ship and $\eta_{j}(j=1,2, \ldots 6)$ is the corresponding motion amplitude ( $\eta_{1}$, surge; $\eta_{2}$, sway; $\eta_{3}$, heave; $\eta_{4}$, roll; $\eta_{5}$, pitch; $\eta_{6}$, yaw); $\eta_{7}=\eta_{0}$ is the incident wave amplitude; $\varphi_{7}$ is the spatial diffraction 
potential; $\varphi_{0}$ is the spatial incident wave potential and $\omega_{e}$ is the encounter frequency, which can be written as

$$
\omega_{e}=\omega-u k \cos \beta
$$

Linear wave theory provides the potential for unit-amplitude incident waves as

$$
\varphi_{0}=-\frac{i g}{\omega} \frac{\cosh k(z+h)}{\cosh k h} e^{i[k(x \cos \beta+y \sin \beta)]}
$$

where $k=\omega^{2} / g$ is the wave number, $\omega$ is the incident wave frequency, $h$ is the water depth, $\beta$ is the angle of wave heading ( $\beta=180 \mathrm{deg}$. corresponds to head sea).

The unsteady perturbation potential $\varphi_{j}$ can be solved by the following boundary value problem:

$$
\nabla^{2} \varphi_{j}=0 \text { in the fluid domain }
$$

$$
\begin{gathered}
-\omega_{e}^{2} \varphi_{j}-2 i \omega_{e} u \frac{\partial \varphi_{j}}{\partial x}+u^{2} \frac{\partial^{2} \varphi_{j}}{\partial x^{2}}+g \frac{\partial \varphi_{j}}{\partial z}=0, \text { on the undisturbed free surface } S_{f} \\
\frac{\partial \varphi_{j}}{\partial z}=0 \text { on the sea bottom } \\
\frac{\partial \varphi_{j}}{\partial n}=\left\{\begin{array}{c}
-i \omega_{e} n_{j}+m_{j}, j=1,2, \ldots, 6 \\
-\frac{\partial \varphi_{0}}{\partial n}, j=7
\end{array}, \text { on the mean wetted body surface } S_{b}\right.
\end{gathered}
$$

The radiation condition at infinity is also imposed to complete the boundary value problem. The generalized normal vectors are defined as

$$
n_{j}=\left\{\begin{array}{c}
\vec{n}, j=1,2,3 \\
\vec{x} \times \vec{n}, j=4,5,6
\end{array}\right.
$$

The $m_{j}$ denotes the $j$-th component of the so-called $m$-term. In the present study, the Neumann-Kelvin linearization can be used to simplify the $m$-terms,

$$
\begin{aligned}
& \left(m_{1}, m_{2}, m_{3}\right)=(0,0,0) \\
& \left(m_{4}, m_{5}, m_{6}\right)=\left(0, n_{3},-n_{2}\right)
\end{aligned}
$$

Furthermore, a Sommerfeld radiation condition with forward speed correction is imposed on the control surface to ensure that the waves can propagate to the far field without reflection.

Once the unknown potential $\varphi_{j}$ are solved, the time-harmonic pressure can be obtained from Bernoulli's equation:

$$
p_{j}=-\rho\left[i \omega_{e} \varphi_{j}+\nabla\left(\varphi_{s}-u x\right) \cdot \nabla \varphi_{j}\right], j=0,1, \ldots, 7
$$

The hydrodynamic force produced by the oscillatory motions of the vessel in the six degrees of freedom can be derived from the radiation potential as

$$
F_{i}^{R}=\sum_{j=1}^{6} \iint_{S_{b}} p_{j} n_{i} d S \cdot \eta_{j}=\sum_{j=1}^{6}\left[\omega_{e}^{2} A_{i j}+i \omega_{e} B_{i j}\right] \eta_{j}, i=1,2, \ldots, 6
$$

where $A_{i j}$ and $B_{i j}$ are the added mass and damping coefficients matrices respectively, which can be written as

$$
\left\{\begin{array}{l}
A_{i j}=\frac{1}{\omega_{e}^{2}} \iint_{S_{b}}\left(u \frac{\partial \varphi_{R j}}{\partial x}-\omega_{e} \varphi_{I j}\right) n_{i} d s \\
B_{i j}=-\frac{1}{\omega_{e}} \iiint_{S_{b}}\left(u \frac{\partial \varphi_{I j}}{\partial x}+\omega_{e} \varphi_{R j}\right) n_{i} d s
\end{array}, \quad i, j=1,2, \ldots, 6\right.
$$


where $\varphi_{R j}$ and $\varphi_{I j}$ is the real part and imaginary part of $j$-th potential. The wave excitation force can be obtained by the integration of incident and diffraction pressure as

$$
F_{i}^{e x t}=\iint_{S_{b}}\left(p_{0}+p_{7}\right) n_{i} d S \cdot \eta_{0}
$$

The wave elevation on the free surface can then be obtained from the dynamic free surface boundary condition in the form

$$
\zeta_{j}=\frac{i \omega_{e}}{g} \eta_{j} \varphi_{j}+\frac{1}{g} \nabla\left(\varphi_{s}-u x\right) \cdot \nabla\left(\eta_{j} \varphi_{j}\right)=\zeta_{R j}+i \zeta_{I j}
$$

where $\zeta_{R j}$ is the real part of $j$-th model, and $\zeta_{l j}$ is the imaginary part.

\section{Numerical implementation}

In the numerical study, the boundary is divided into a number of quadrilateral panels with constant source density $\sigma_{(\xi)}$, where $\xi=(\xi, \eta, \zeta)$ is the position vector on the boundary. If $\mathbf{x}=(x, y, z)$ is inside the fluid domain or on the boundary surface, the potential can be expressed by a source distribution on the boundary of the fluid domain:

$$
\varphi(\vec{x})=\iint_{S_{b}+S_{f}+S_{c}} \sigma(\vec{\xi}) G(\mathbf{x}, \boldsymbol{\xi}) d S_{\xi}
$$

where $\varphi$ denotes the steady potential $\varphi_{s}$ or the unsteady potential $\varphi_{j}, G_{(\mathbf{x}, \xi)}$ is the Rankine-type Green function that satisfies the sea bed boundary condition through the method of mirror image:

$$
G(\mathbf{x}, \boldsymbol{\xi})=\frac{1}{\sqrt{(x-\xi)^{2}+(y-\eta)^{2}+(z-\varsigma)^{2}}}+\frac{1}{\sqrt{(x-\xi)^{2}+(y-\eta)^{2}+(z+2 h+\varsigma)^{2}}}
$$

If we have $N$ panels on the body surface, free surface and control surface together, the potential in point $\mathbf{x}$ becomes

$$
\varphi\left(\mathbf{x}_{i}\right)=\sum_{j=1}^{N} \frac{\sigma_{j}}{4 \pi} \iint_{S_{b}+S_{f}+S_{c}} G\left(\mathbf{x}_{i}, \xi\right) d S_{\xi}=\sum_{j=1}^{N} \frac{\sigma_{j}}{4 \pi} G_{i, j}
$$

When the collocation point and the panel are close to each other, the influence coefficients $G_{i, j}$ can be calculated with analytical formulas listed by Prins (1995) when the distance between the collocation point and the panel is large, these coefficients are calculated numerically. The same procedure can be applied to discretize the boundary integral for the velocity

$$
\frac{\partial \varphi}{\partial n}\left(\mathbf{x}_{i}\right)=\frac{1}{2} \sigma_{i}+\sum_{\substack{j=1 \\ j \neq i}}^{N} \frac{\sigma_{j}}{4 \pi} \iint_{S_{b}+S_{f}+S_{c}} \frac{\partial}{\partial n_{i}} G\left(\mathbf{x}_{i}, \boldsymbol{\xi}\right) d S_{\xi}=\frac{1}{2} \sigma_{i}+\sum_{\substack{j=1 \\ j \neq i}}^{N} \frac{\sigma_{j}}{4 \pi} G_{i, j}^{n}
$$

The analytical formulas of the influence coefficients $G_{i, j}^{n}$ are listed by Hess and Smith(1964).

The singularity distribution does not have to be located on the free surface itself, it can also be located at a short distance above the free surface, as long as the collocation points, where the boundary condition has to be satisfied, stay on the free surface. In practice, a maximal distance of three times the longitudinal size of a panel is possible (Bunnik, 1999). In the present study, the raised distance $\Delta z_{i}=\sqrt{S_{i}}$, where $S_{i}$ is the area of the $i$-th panel.

Special attentions should be paid on the second derivative of the potential on the free surface. Generally, the difference schemes can be divided in two classes: up wind difference schemes and central difference schemes. Although central difference schemes are supposed to be more accurate, the stabilizing properties of the upwind difference schemes are more desired in the forward speed problem (Bunnik, 1999). Physically this can be explained by the fact that new information on the wave pattern mainly comes from the upstream side, especially at high speeds, whereas the downstream side only contains 
old information. The second-order upwind difference scheme for the second derivative of the potential to $x$ can be written as follows

$$
\frac{\partial^{2} \varphi}{\partial x^{2}}\left(\vec{x}_{i}\right)=\frac{1}{\Delta x^{2}}\left[\frac{1}{4} \varphi\left(\vec{x}_{i+4}\right)-2 \varphi\left(\vec{x}_{i+3}\right)+\frac{11}{2} \varphi\left(\vec{x}_{i+2}\right)-6 \varphi\left(\vec{x}_{i+1}\right)+\frac{9}{4} \varphi\left(\vec{x}_{i}\right)\right]
$$

\section{Results and discussions}

The above theory is applied to our in-house developed 3D BEM program MHydro to investigate the side wall effects both in calm water and waves.

\subsection{Side wall effects on experiments in calm water}

\subsubsection{Validations}

Before large numerical calculations were performed, a rigorous validation of the numerical program must be conducted. For the wave-making problem, the validations are established on the open water tests of a Wigley III hull due to the fact that there are considerable experiments data available. The ship model can be defined as:

$$
y=\frac{B}{2}\left[1-\left(\frac{z}{D}\right)^{s}\right]\left[1-\left(\frac{2 x}{L}\right)^{2}\right]\left[1+0.2\left(\frac{2 x}{L}\right)^{2}\right]
$$

The main dimensions of Wigley III model are shown in Table 1. Fig. 2 shows the panel distribution on the computational domain.

\begin{tabular}{ll}
\multicolumn{2}{c}{ Table 1. Main dimensions of Wigley III hull } \\
\hline Length, $L(m)$ & 3 \\
Breadth, $B(m)$ & 0.3 \\
Draught, $D(m)$ & 0.1875 \\
Displacement, $V\left(m^{3}\right)$ & 0.078 \\
Centre of rotation above base, $K R(m)$ & 0.1875 \\
Centre of gravity above base, $K G(m)$ & 0.17 \\
Radius of inertia for pitch, $k_{y y}(m)$ & 0.75
\end{tabular}

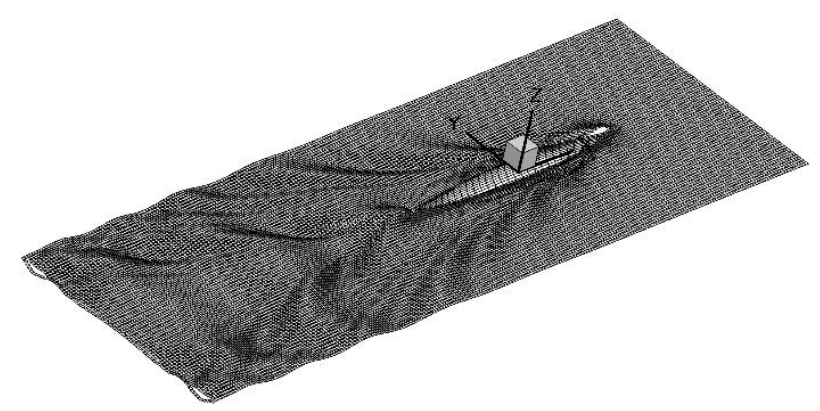

Fig. 2. The coordinate system and panel distribution on the computational domain of a Wigley III hull advancing in open calm water. There are 9,900 panels distributed on the half computational domain: 300 on the body surface and 9,600 on the free surface. The computational domain is truncated at $L$ upstream and $2 L$ downstream. The figure also shows the waves produced by Wigley hull at $F_{n}=0.3$, where $F_{n}=u / \sqrt{g L}$ is the Froude number. 


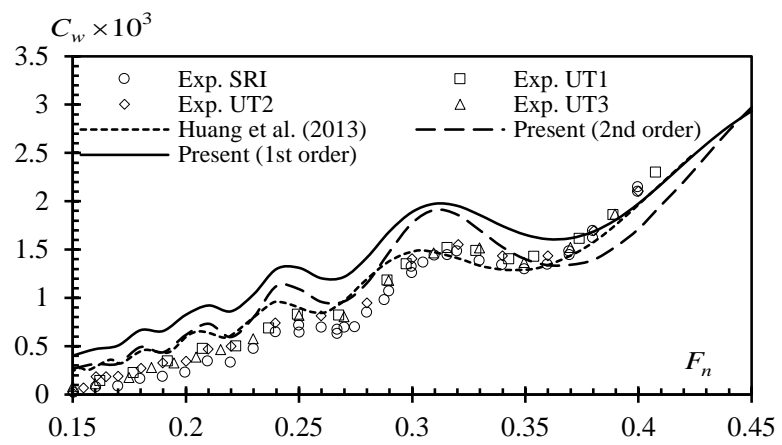

Fig. 3. Wave-making resistance coefficient of a Wigley hull. The experiments are conducted by Ship Research Institution (SRI), University of Tokyo (UT).

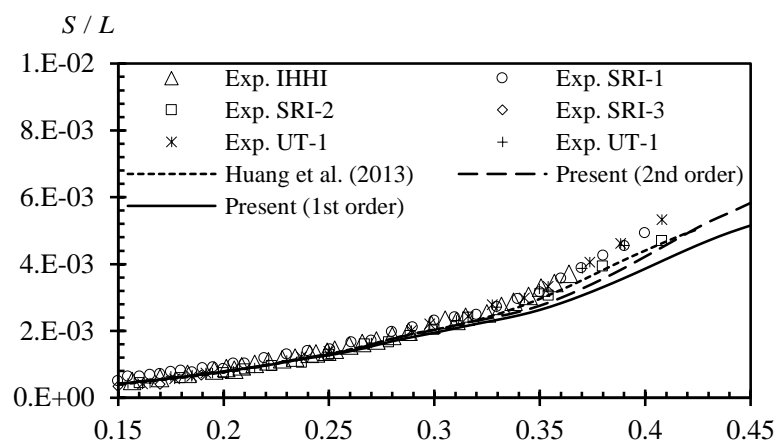

Fig. 4. Sinkage of a Wigley hull. IHHI indicates the experiments conducted by Ishikawajim-Harima Heavy Industries Co., Ltd..

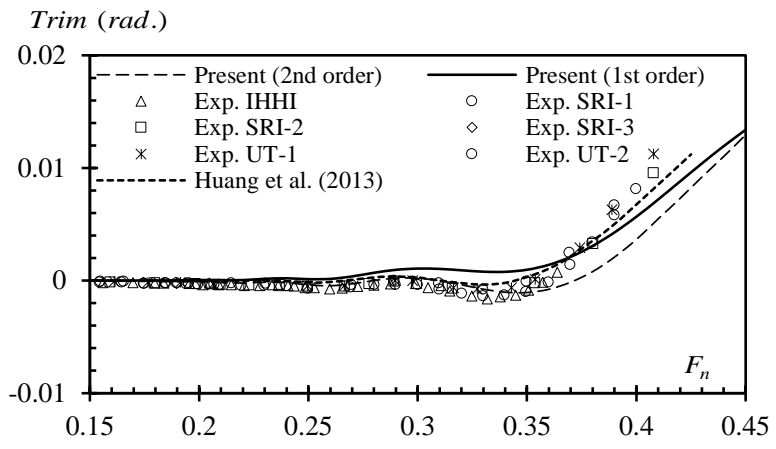

Fig. 5. Trim of a Wigley hull.

Fig. 3 shows the wave-making resistance results of a Wigley hull. The experimental data presented in the figure was obtained by subtracting the frictional component from the measured total resistance. Schoenherr formula was employed to calculate the frictional drag, and the three dimensional form factor on flat plate skin friction was 0.095 for SRI, 0.045 for UT1, and 0.047 for UT2 and UT3. Both $1^{\text {st }}$ order and $2^{\text {nd }}$ order results are included in Fig. 3. The $1^{\text {st }}$ order results are obtained by solving BVP from Eq. (3), (4), (8) and (12). The $2^{\text {nd }}$ order results are obtained by solving BVP from Eq. (3), (5), (9) and (12). The numerical results from Huang et al. (2013) obtained from Neumann-Michell theory as well as the experimental measurements from different institutions (Kajitani, 1983) are also included in the comparisons. Generally, the present program MHydro has a satisfactory prediction of the wave-making resistance. The $1^{\text {st }}$ order solution overestimates the wave-making resistance at $F_{n}<0.35$, and at this range of Froude number, the $2^{\text {nd }}$ order solution provides a better estimation. Regarding the sinkage and trim (as shown in Fig. 4 and Fig. 5), the agreement between the present calculations and the experimental results are very well. The discrepancy becomes evident as the forward speed increases to $F_{n}>0.35$. This is mainly due to the body surface boundary condition. As the speed of ship increases, the wave elevation increases significantly. At high speed case, the large wave elevation could modify the total wet surface of the ships. Therefore, the body surface boundary in Eq. (3), (4) cannot be satisfied 
on the mean wet surface. An instant wet body surface must be taken into consideration to account the nonlinear effects. The discussion about the nonlinearity of the body surface boundary condition can be found in Chen et al. (2016). In the present study, we aim to discuss the side wall effects and no attempt is made to discuss the nonlinearity of the body surface.

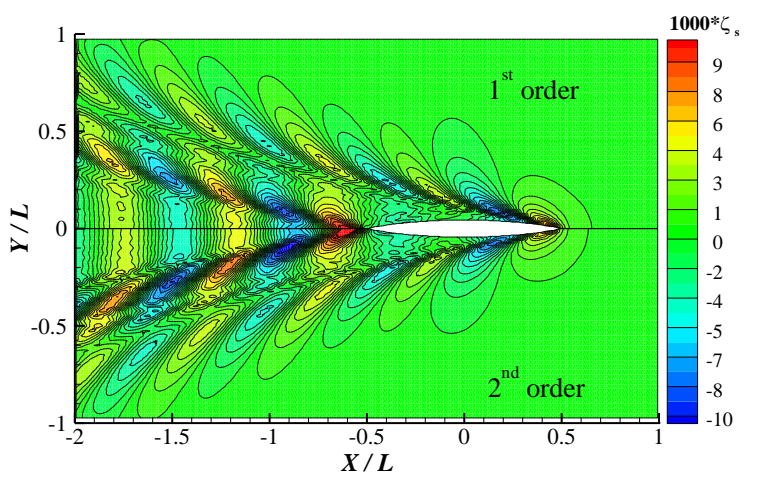

Fig. 6. Steady wave pattern produced by a Wigley hull in open and deep water at $F_{n}=0.3$.

Fig. 6 is the steady wave pattern produced by a Wigley hull in open and deep water at $F_{n}=0.3$. The upper half of Fig. 6 represents the wave pattern obtained by using the $1^{\text {st }}$ order free surface boundary condition, while the lower half of the figure shows the $2^{\text {nd }}$ order results. Fig. 7 shows the wave profiles along a Wigley hull with different forward speeds. The overall agreement between the present predictions and experimental results is very satisfactory. The discrepancies occur at the bow and stern parts, where the stagnation points are located. The underestimations were attributed to the nonlinear effects. Even though the present calculation is performed up to 2nd order, the higher-order nonlinear effects are essential for the predictions of the waves at ship bow area. Raven (1994) proved that the underestimation of diverging waves was caused by neglecting nonlinear effects. And including nonlinear terms would consistently improves the results.

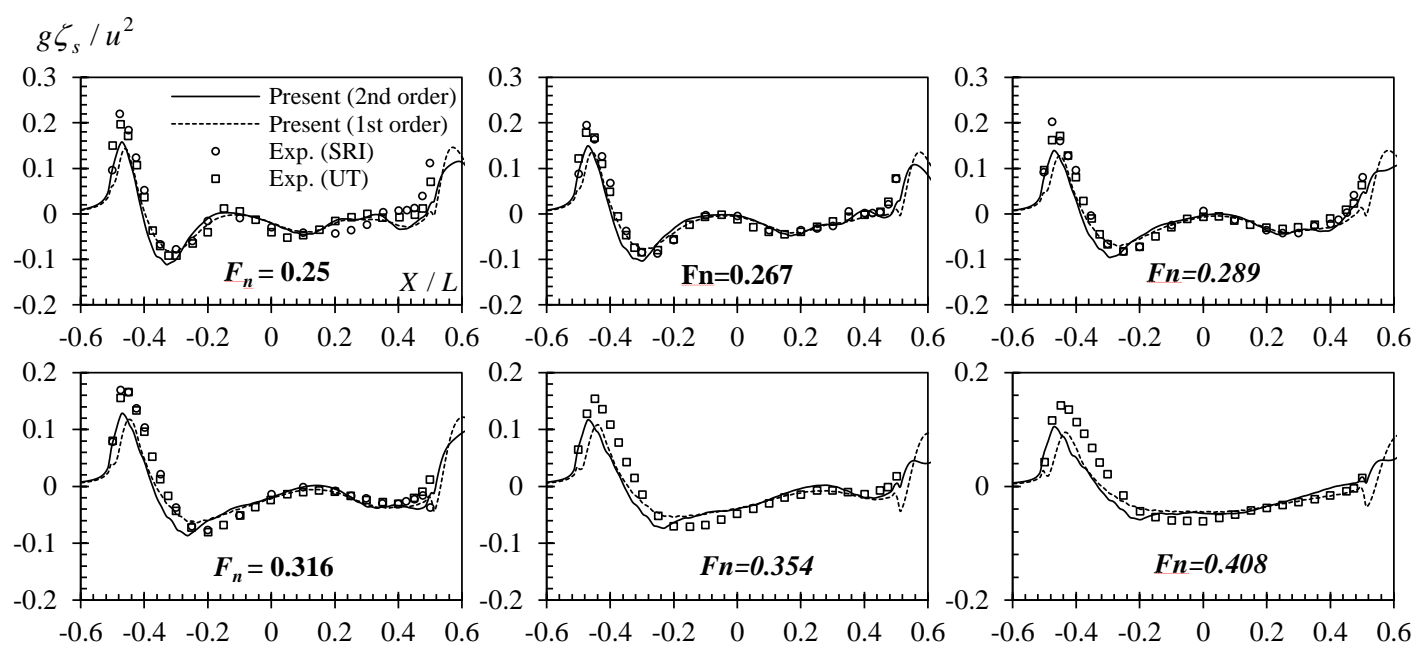

Fig. 7. Wave profiles along a Wigley hull at different Froude numbers.

\subsubsection{Side wall effects at deep water}

In order to simulate the side wall effect, we distribute source panels on the side walls. The side wall boundary condition in Eq. (12) is applied. Fig. 8 compares the deep water results of wave-making resistance coefficients, sinkage and trim at a wide range of $w / L$. It can be concluded from Fig. 8 that at $F_{n}<0.35$, the side wall effects can be neglected at $w / L>0.6$ in deep water. However, when conducting high speed model tests at $F_{n}>0.35$, the width of the tank is required to be larger and the side wall effects can be neglected at $w / L>0.8$. It can also be found the side wall effects are significant at $w / L=0.4$. This is inconsistent with the theoretical estimation from Eq. (1), where the side wall effects are estimated to be vanished at $w / L>0.36$. This is due to the near field local waves produced by the 3D ship, as shown in Fig. 9. 
(a)

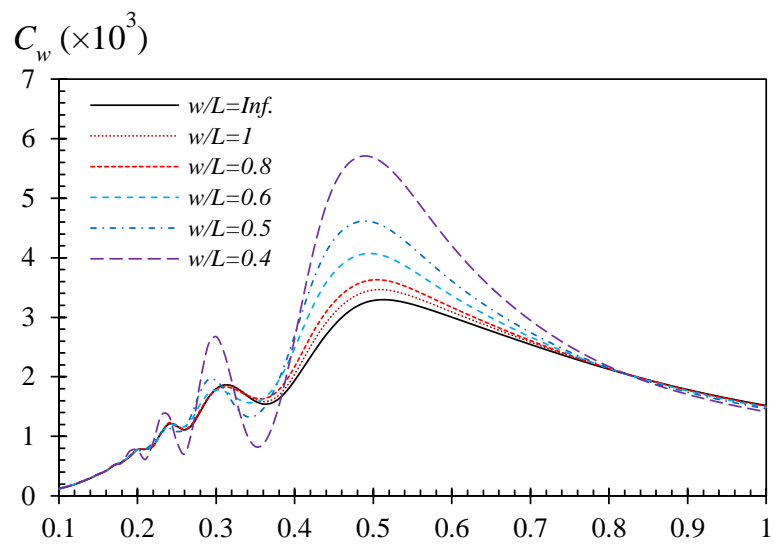

(b)

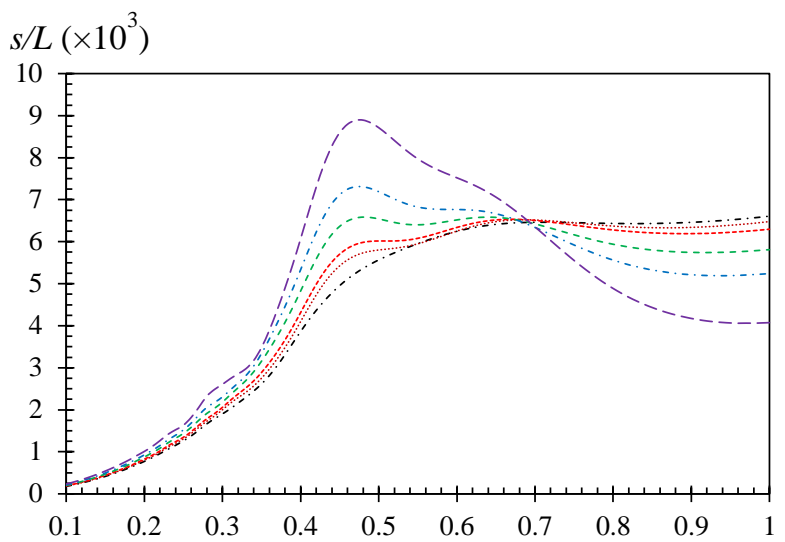

(c)

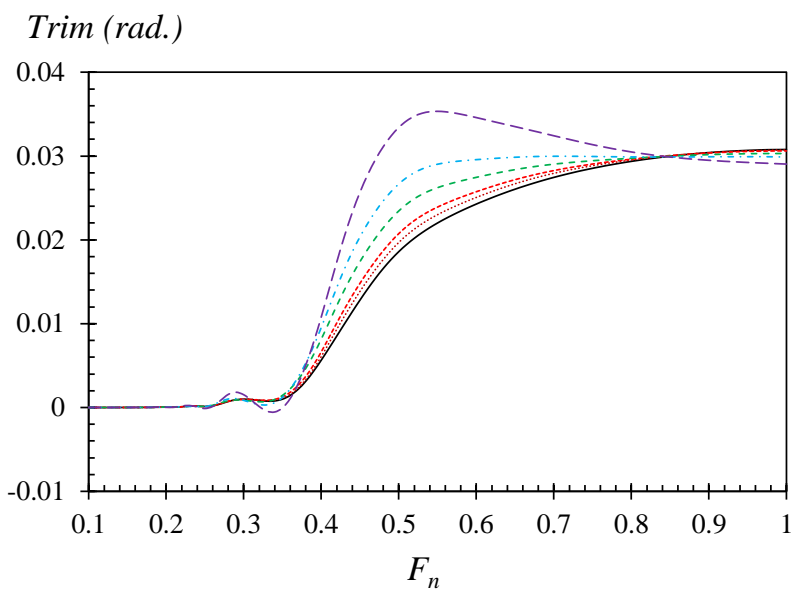

Fig. 8. Side wall effects on (a) wave-making resistance coefficients; (b) sinkage; (c) trim.

Fig. 9 (a) compares the wave elevation at $w / L=1$ to the open water results with the same Froude number at $F_{n}=0.3$. At the range of $X / L>-0.6$, the difference between open water results and the results with side walls can hardly be found. The Kelvin waves propagate to the far field downstream and strike the side wall at around $X / L=-1$, where a reflection occurs. The reflected waves modify the flow field at $X / L<-1$, where they can never strike the ship model. Therefore, the side wall effects can be neglected at $w / L=0.5$. However, as shown in Fig. 9 (b), as the breadth of the tank becomes very small ( $w / L=0.2)$, the wave field is completely modified by the reflected waves from the side walls. At $w / L=0.2$, the waves produced by the bow propagate downstream and the first trough strikes the side wall at around $X / L=0.05$. The subsequent reflected waves (the trough) strike the stern of the ship, which reduces the pressure distributed on the stern. Therefore, the difference of the pressure on the bow and stern becomes evident and the wave-making resistance, as well as the trim, will be subject to 
increase at $F_{n}=0.3$, as shown in Fig. 8 (a) and (c). However, it cannot be concluded that the wavemaking resistance or trim will increase as the breadth becomes smaller. It also depends on the speed. As can be seen from Fig. 9 (c), at $F_{n}=0.35$, the crest of the reflected wave strikes the stern, which enlarges the pressure distribution in the stern area. Therefore, the difference of the pressure on the bow and stern becomes smaller. The wave-making resistance and the trim at $F_{n}=0.35$ become smaller than those in open water.

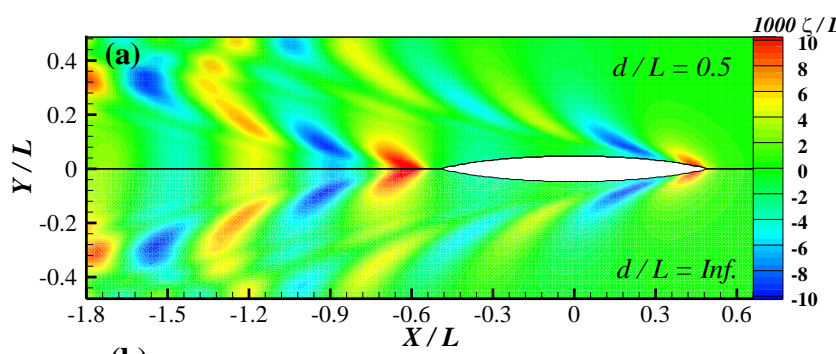

(b)
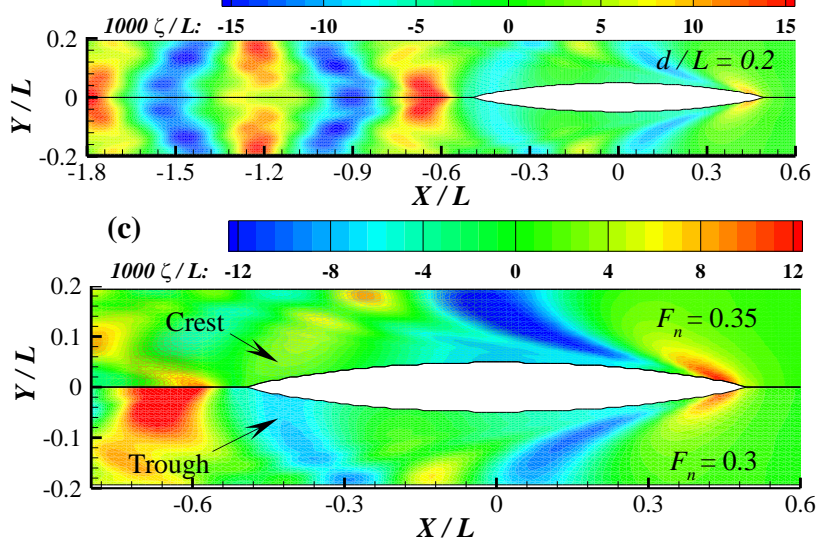

Fig. 9. Wave elevation on the free surface. (a) Upper half of the figure shows the wave elevation at $w / L=$ 1 with Froude number at $F_{n}=0.3$; lower half of the figure shows the wave elevation in open water with Froude number at $F_{n}=0.3$; (b) wave elevation at $w / L=0.4$ with Froude number at $F_{n}=0.3$; (c) Upper half of the figure shows the wave elevation at $w / L=0.4$ with Froude number at $F_{n}=0.35$; lower half of the figure shows the wave at $w / L=0.4$ with Froude number at $F_{n}=0.3$.

\subsubsection{Diagram of side wall effects in calm water}

The water depth of the tank $h$, which can be nondimensionalized by ship draught $D$, could amplify the side wall effects when conducting model testing. In the present study, the wave-making resistance coefficient $\left(C_{w}\right)$ is used as a reference to estimate the side wall effects. Fig. 10 shows the side wall effects at $h / D=10$. In fact, for each $h / D$, we can always obtain a group of $C_{w}$ curves with various $w / L$ values, which can be denoted by $C_{w}^{(d / L)_{n}}$. The side wall effects then can be expressed as

$$
E_{s}=\left|\frac{C_{w}^{(d / L)_{n}}-C_{w}^{I n f}}{C_{w}^{I n f}}\right| \times 100 \%
$$

where $C_{w}^{\text {Inf }}$ represents the results without side wall effects $(w / L=I n f$.$) , and the subscript n$ is an identifier of $w / L$, for example, $n=1$ corresponds to $(w / L)_{1}=0.4$. For each $w / L$ curve, the maximum side wall effect $E_{s}^{\max }$ can be calculated. Varying $w / L$ from 0.2 to 10 with a step increasing at 0.1 , a curve of $E_{s}^{\max }$ can be obtained. Repeating the above process for a series of $h / D$, a group of $E_{s}^{\max }$ curves can be depicted, as shown in Fig. 11. We only display limited curves in Fig. 10 and Fig. 11, where $w / L$ varies from 0.2 to 10 , since the width of tanks $(w)$ in most towing tanks could not exceed $10 L$. The maximum side wall effect $E_{s}^{\max }$ is truncated at $50 \%$. The Froude number $F_{n}$ varies from 0.1 to 1 , which covers most of the speed range when conducting a ship model testing. It should be noted that in finite water depth, the critical or near critical speeds $\left(F_{h} \rightarrow 1\right)$ are also covered in the present calculations. Theoretically, the flow at $F_{h} \rightarrow 1$ should be unsteady and solitary waves will be generated, propagating upstream ahead of the ship (Chen and Sharma, 2006; Li and Sclavounos, 2002). Therefore, the present assumption of 
steady flow and the numerical treatment of second-order upwind scheme is not adequate to predict the hydrodynamic features when the ship is travelling at a near-critical speed. However, in the present study of side wall effects, the extreme shallow water cases are not essential. As can be seen from Fig. 11, the side wall effect is greater than $15 \%$ at $h / D=6$, even though the width of the tank is 10 times as large as the ship length. It can also be found from Fig. 10 that wave-making resistance curves are very smooth and there is no sudden drops near critical speeds $\left(F_{h} \rightarrow 1\right)$. The maximum side wall effect $E_{s}^{\max }$ is observed at $F_{h} \rightarrow 0.6$, rather than at critical speed. Therefore, the present calculation is still reliable in the prediction of side wall effects.

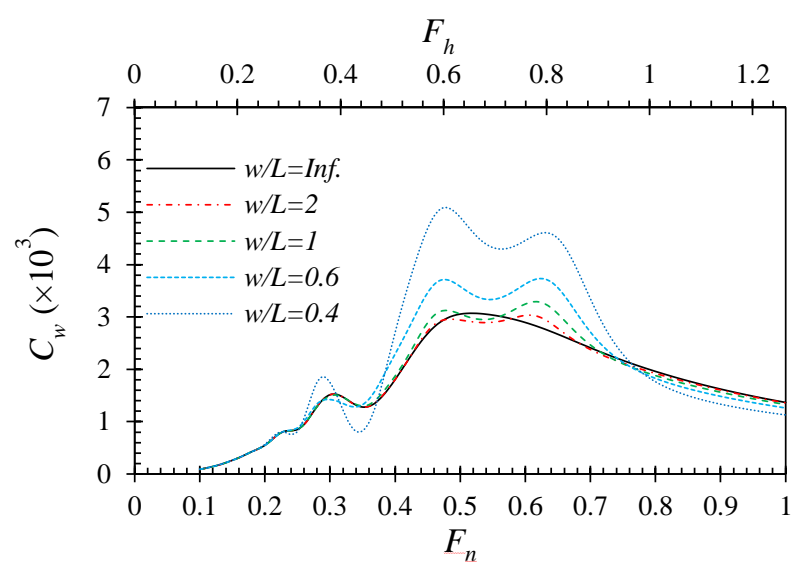

Fig. 10. Side wall effects on wave-making resistance coefficient at $h / D=10$.

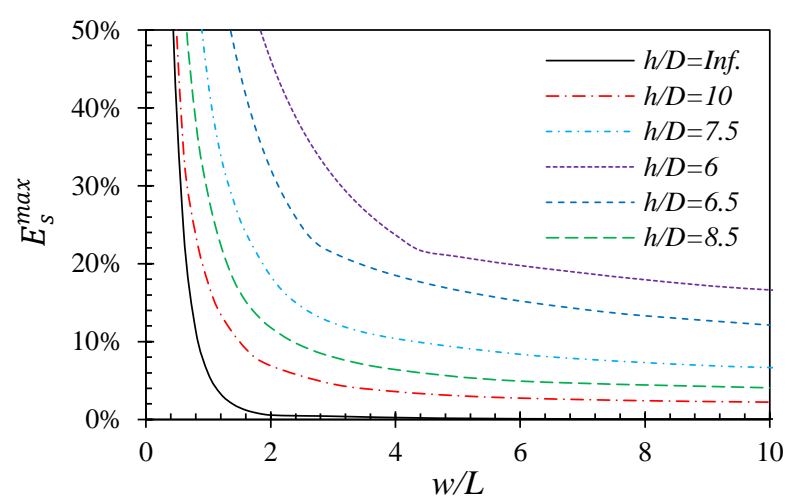

Fig. 11. Maximum side wall effect

In the numerical calculations, the simulation matrix (including two parameters, $w / L$ and $h / D$ ) must be adequate to generate smooth curves shown in Fig. 11 and Fig. 12. Since the calculations are based on potential flow solver, the computation is not so time-consuming. Therefore, it is desirable to prepare a diagram to describe the side wall effects at various water depths and tank widths. A large number of computations were performed by a combination of $w / L$ and $h / D$. Then we can draw a series of curves, which are used to describe that the side wall effect is less than the permissible error to be included in the measured values. These curves are shown in Fig. 12. From Fig. 12, we can estimate the side wall effects of a towing tank at any given width and depth. For example, in a ship model testing, the water depth and width of the tank is measured at $h / D=10$ and $w / L=1$. Then the maximum side wall effect can be estimated to be $5 \%<E_{s}^{\max }<10 \%$. Of course, the maximum side wall effect mentioned here corresponds to the Froude number varying from 0 to 1 . If the maximum Froude number is reduced to 0.3 (low and mediate speed), the side wall effect will be smaller. Alternatively, the diagram shown in Fig. 12 can also be used to control side wall effects in a testing. For a towing tank, the width is tank is usually fixed, while the water depth can be adjusted. For a tank with $w / L=2$, in order to sustain the maximum side wall effect $E_{s}^{\max }$ under 5\%, the water depth must be greater than that of ship draught $(h / D>11.2)$. It is impossible to sustain the maximum side wall effect $E_{S}^{\max }$ under $5 \%$ if $h / D<8$, no matter how wide the tank is. 


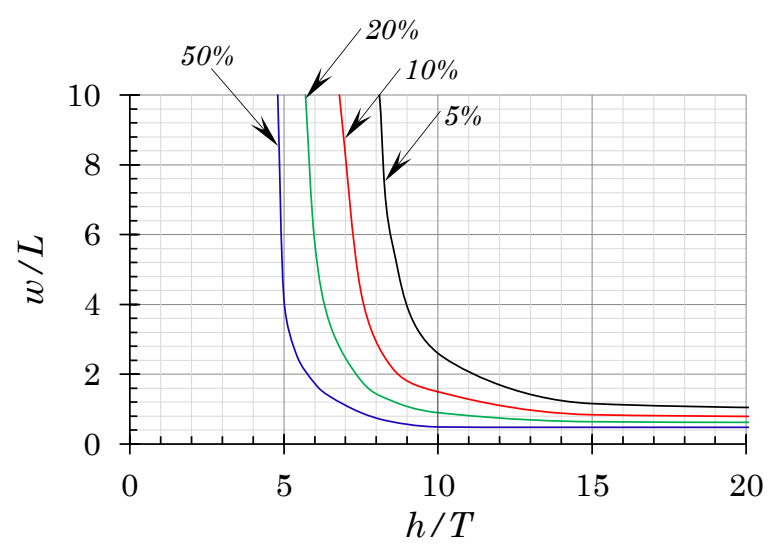

Fig. 12. Diagram showing the side wall effects with various permissible errors

\subsection{Side wall effects on experiments in waves}

\subsubsection{Validations}

The side wall effect on experiments in waves are more complicated than that in calm water. According to the asymptotic far field wave theory of a translating and oscillating singularity, there are at least three individual wave systems existing on the free surface, rather than a single Kelvin wave in calm water. It is well known that the far field patterns are determined by parameter $\tau$ (Noblesse and Hendrix, 1992; Yuan et al., 2015b). A critical value of $\tau$ is $1 / 4$, where the wave group travels the same speed with the singularity. In the previous studies, the difficulties arise from the proper radiation condition on the control surfaces at $\tau<1 / 4$. We complemented a new radiation condition to the classical boundary value problem, which enables us to investigate the waves produced by a translating and oscillating object at $\tau<1 / 4$ (Yuan et al., 2014a; Yuan et al., 2014b). In the present study, this radiation condition will be used to investigate the side wall effects on experiments in waves. Before conducting massive numerical calculations, a rigorous validation of the numerical program should be conducted. Kashiwagi and Ohkusu's (1991) model test results will be used here to validate the numerical program.

The model used here is a Lewis-form ship and its principal particulars are shown in Table 2. The model test was conducted in the towing tank (60 $\mathrm{m}$ length, $4 \mathrm{~m}$ breadth, $2.3 \mathrm{~m}$ in depth) of the Nagasaki Institute of Applied Science (Kashiwagi and Ohkusu, 1991). The model was advancing at a Froude number $F_{n}$ $=0.1$ in the waterway of $w / L=2.67$. In the numerical calculation, 4,180 panels are distributed on the half domain of the symmetrical boundaries (360 panels on the body surface, 2,700 on the free surface, 640 on the side walls and 480 on the control surfaces), as shown in Fig. 13. The free surface is truncated at $L$ upstream and $2 L$ downstream.

Table 2. Main dimensions of Lewis-form ship

\begin{tabular}{ll}
\hline Length, $L(m)$ & 1.5 \\
Breadth, $B(m)$ & 0.25 \\
Draught, $D(m)$ & 0.125 \\
Block coefficient, $C_{B}$ & 0.659 \\
Midship section coefficient, $C_{M}$ & 0.1875 \\
Waterplane area coefficient, $C_{A}$ & 0.17
\end{tabular}




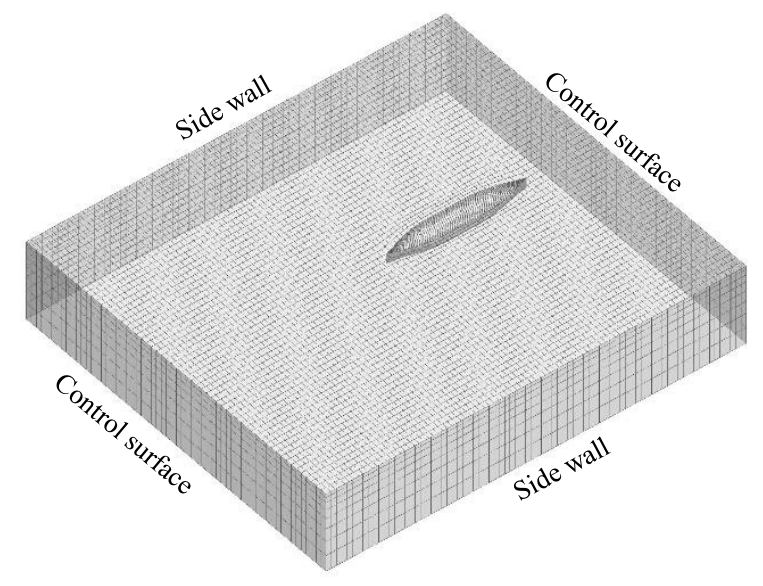

Fig. 13. Panel distribution on a Lewis-form ship advancing in a towing $\operatorname{tank}$ of $w / L=2.67$.

Fig. 14 and Fig. 15 show the validations of hydrodynamic coefficients. In general, the present calculations from MHydro have a satisfactory agreement with the experimental measurements as well as the numerical results based on slender ship theory. For some components, the traditional slender ship theory could even provide a better prediction. As the parameter $\tau<0.25$ (or $\omega_{e} / \sqrt{L / g}<2.5$ ), the hydrodynamic coefficients (radiation forces) fluctuate violently away from the open sea results. This is due to the reflected waves that strike the ship model. It can also be observed that the agreement between present calculations and experiments is very satisfactory even at parameter $\tau<0.25$ (or $\omega_{e} / \sqrt{L / g}<2.5$ ), which indicates the radiation condition included in the present numerical program MHydro is capable of predicting the hydrodynamic properties of the advancing ships, even at parameter $\tau<0.25$ (or $\omega_{e} / \sqrt{L / g}<2.5$ ). As the nondimensional frequency increases, the hydrodynamic coefficients gradually approach the open sea results and the side wall effects tend to diminish. Fig. 16 shows the wave exciting forces of a Lewis-form ship of $B / L=1 / 6$ in waterway of $w / L=2.67\left(F_{n}=0.1\right)$. Both the heave force and pitch moment agree well with the experimental measurements and the published numerical results based on slender ship theory (Kashiwagi and Ohkusu, 1991). A very large spike can be observed at $\lambda$ / $L=1.47$, which corresponds to $\tau=0.25$. It can be found from Yuan et al. (2016) that as the parameter $\tau$ increases, the semi-wedge angle becomes smaller. Therefore, the reflecting point shifts downstream and less reflected waves can strike the ship model. The steady wave system is a special case of parameter $\tau=0$, when the speed is nonzero while the oscillating frequency is zero. In this case, the unsteady problem in waves reduces to steady problem in calm water.
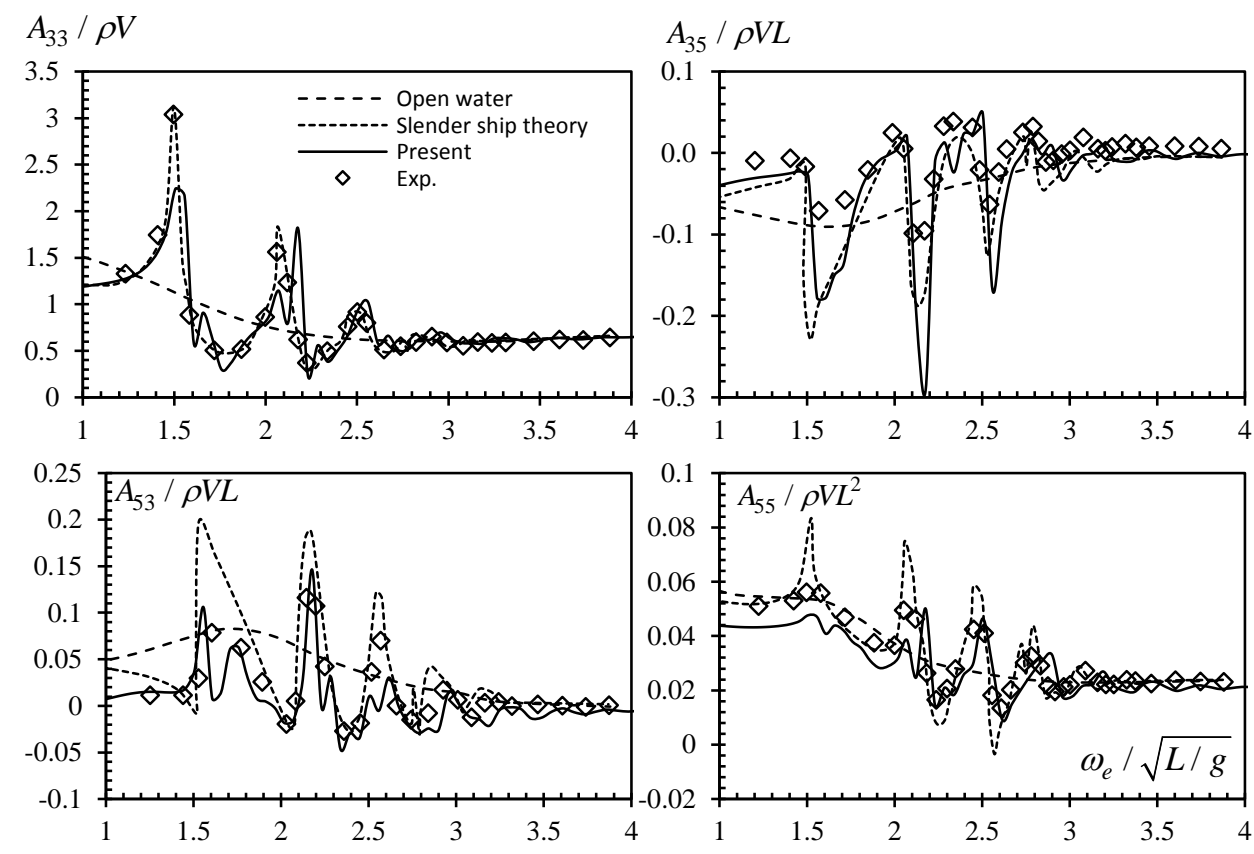
Fig. 14. Added mass of a Lewis-form ship of $B / L=1 / 6$ in waterway of $w / L=2.67\left(F_{n}=0.1\right)$. The slender ship results and experiments were provided by Kashiwagi and Ohkusu (1991).
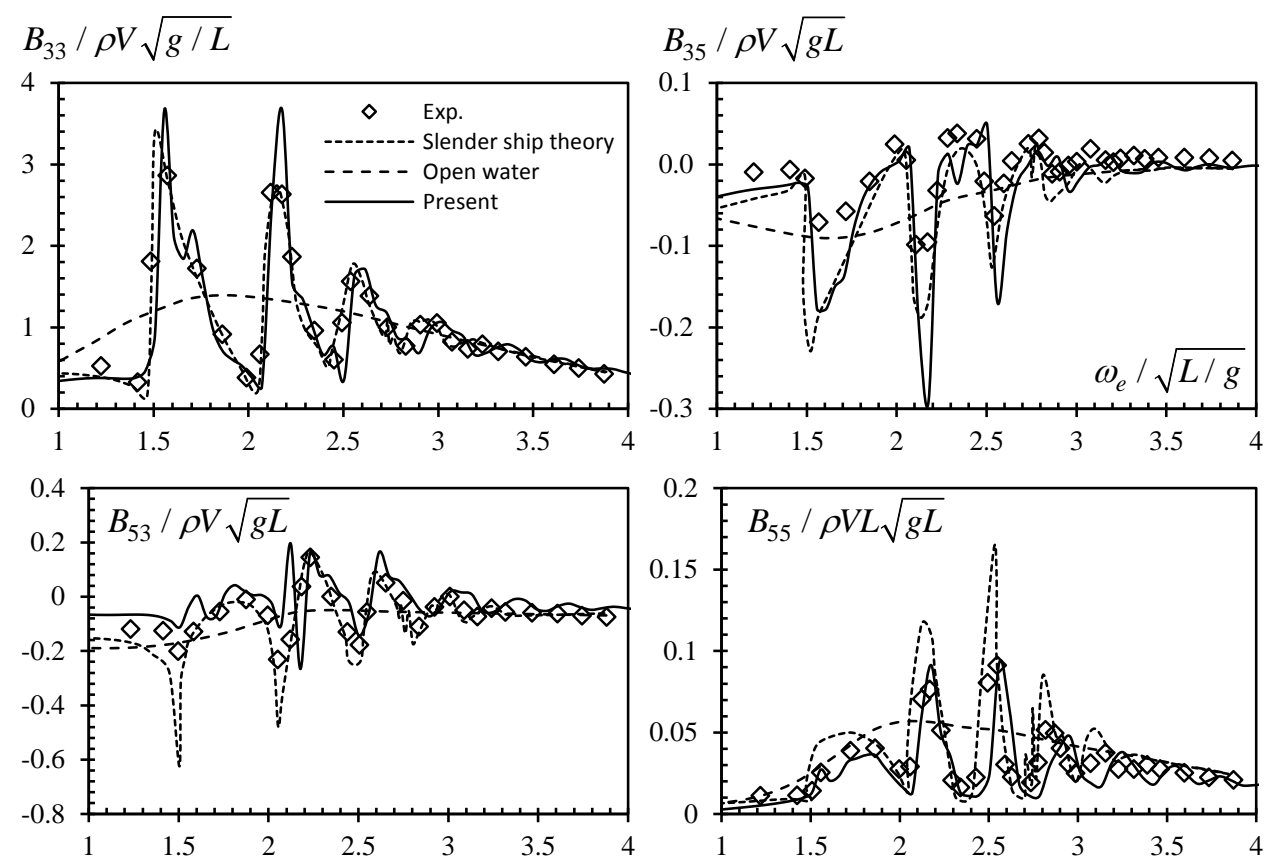

Fig. 15. Damping of a Lewis-form ship of $B / L=1 / 6$ in waterway of $w / L=2.67\left(F_{n}=0.1\right)$.

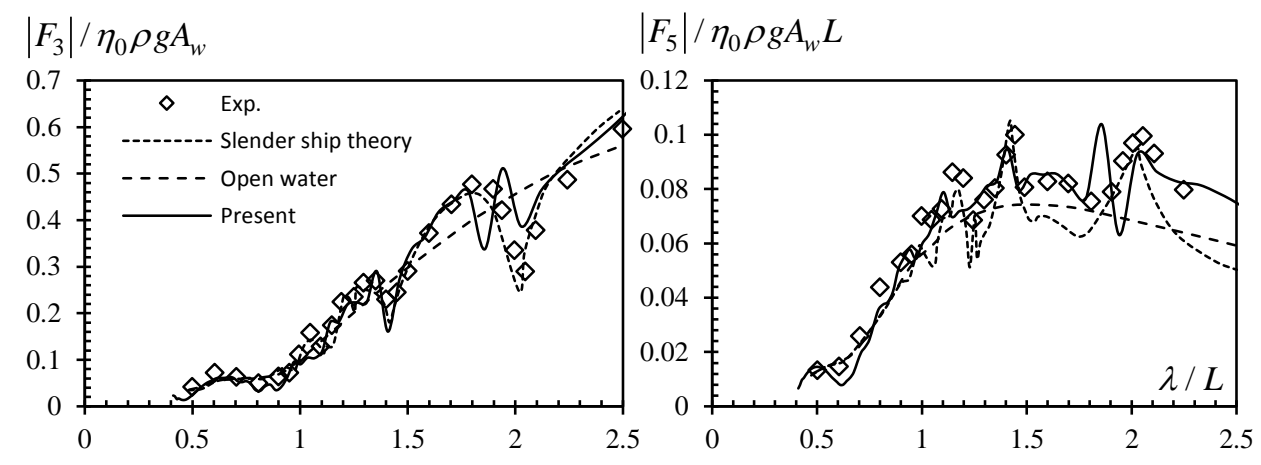

Fig. 16. Wave exciting forces of a Lewis-form ship of $B / L=1 / 6$ in waterway of $w / L=2.67\left(F_{n}=0.1\right)$.

As described above, a jump of side wall effects was observed at critical parameter $\tau=0.25$. As $\tau<0.25$, the scattered waves could travel ahead of the ship model. In this case, the reflections from the side walls are inevitable. As $\tau$ increases, the scattered waves will be confined within a smaller wedge angle. The relationship between $\tau$ and semi-wedge angle can be found in Yuan, et al. (2016). In order to explore the reflected waves from the side walls, two typical values of $\tau$ are examined: $\tau=0.2$ and $\tau=0.5$. The wave patterns at $\tau=0.2$ and $\tau=0.5$ are shown in Fig. 17 and Fig. 18. At $\tau=0.2$, a very interesting phenomenon was observed: the reflected waves are trapped in the towing tank and they cannot propagate downstream (as shown in Fig. 17). These reflected waves of course will strike the ship model and therefore affect the hydrodynamics of the ship model significantly. At $\tau=0.5$, the scattered waves are confined within a smaller semi-wedge angle (as shown in Fig. 18). The scattered waves produced at ship bow approach the side wall at point $P_{1}$, where the reflection occurs. The reflected waves propagate to point $P_{2}$, which is far away from the ship model. In this case, the reflections from the side walls could not modify the flow field around the ship model. As can be seen from Fig. 18, the wave elevation around the ship model with side walls is almost the same to that without side walls. This explains why the side wall effects diminish as $\tau\left(\right.$ or $\left.\omega_{e}\right)$ increases (see Fig. 14 and Fig. 15). 


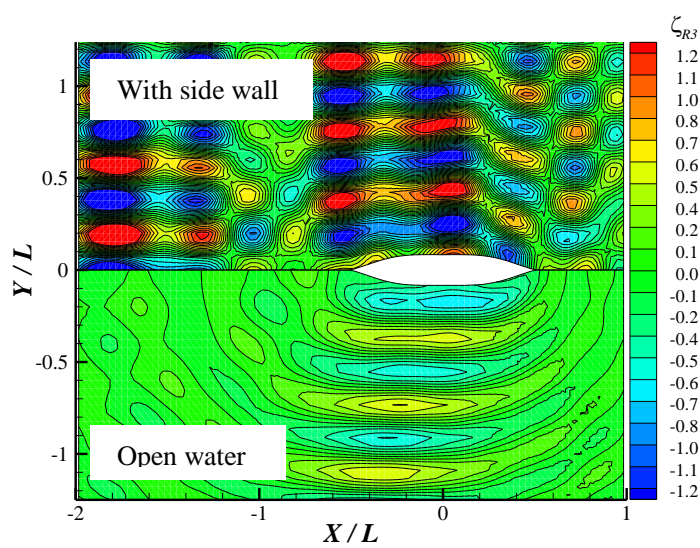

Fig. 17 Real part of radiated waves for unit heave motion of a Lewis-form hull of $B / L=1 / 6$ in waterway of $w / L=2.67\left(F_{n}=0.04, \tau=0.2\right)$. Upper half of the figure shows the wave elevation with side wall effects; lower half of the figure shows the results in open water.

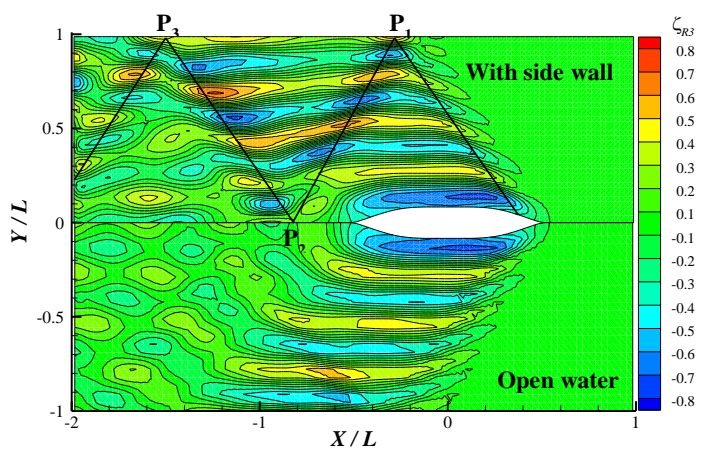

Fig. 18 Real part of radiated waves for unit heave motion of a Lewis-form hull of $B / L=1 / 6$ in waterway of $w / L=2.67\left(F_{n}=0.1, \tau=0.5\right)$. The auxiliary lines in the upper half of the figure represent the scattered and reflected wave routes.

\subsubsection{Diagram of side wall effects in waves}

Theoretically, the resonant modes in the gap between the ship model and side walls are essential for the estimation of the side wall effects (Zhao et al., 2017). However, in the present study, we are particularly interested in the high-frequency range where the side wall effects are not significant. As can be seen from Fig. 14 to Fig. 16, the amplitudes of the resonant modes decreases rapidly when the frequency increases, which indicates the side wall effects will diminish gradually as the nondimensional frequency increases to a certain value. During the calculation, we find that for any given Froude numbers, we can always find a critical frequency showing the existence of the side wall effects. Therefore, we can determine the critical curves showing the existence of the side wall effects as a function of Froude number, frequency and transverse distance. A diagram of these curves was originally presented by Yuan, et al. (2016) to illustrate the hydrodynamic interaction effects between two travelling ships. Theoretically, the side wall effects problem can be transformed to a ship-to-ship problem by using the method of mirror image, which has been widely used to deal with the sea bottom boundary condition. Mathematically, the impenetrable condition on the side walls is similar to the sea bottom condition. The existence of the side wall 2 can be replaced by putting a mirror there, as shown in Fig. 19. Therefore, the same diagram, which was presented to illustrate the hydrodynamic interaction effects, can be used in the present study to show the side wall effects, as shown in Fig. 20. 


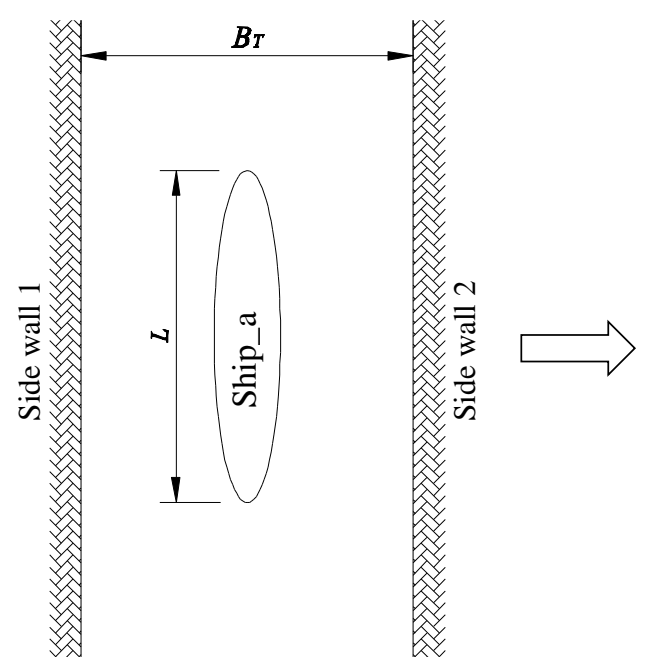

(a)
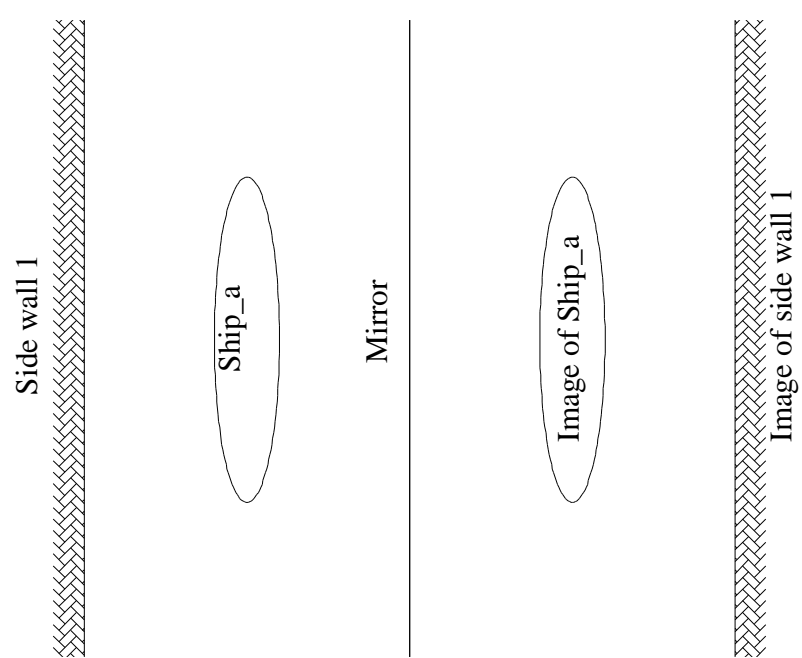

(b)

Fig. 19. Sketch of side wall effects and ship-to-ship problem

The $x$-axis in Fig. 20 is $\sqrt{g / L} / \omega_{e}=F_{n} / \tau, y$-axis is $F_{n}$. The ratio of $y$ to $x$ represents the parameter $\tau$. In the present numerical calculation, for a given value of $w / L$, the critical parameter $\tau$ is a unique constant value. Therefore, the dashed lines in Fig. 20 are linear and they represent the critical line estimated from the asymptotic far-field wave theory (Yuan et al., 2016). The solid curves are the calculated critical lines, which approach the dotted lines at high frequency, where the wavelength is relatively small compared to the transverse distance and the theoretical estimation is valid. As the encountered frequency decreases, the discrepancies become evident and the range of side wall effects expands. The difference between the dashed lines and solid curves is due to the effect of the near-field non-radiation local waves in the vicinity of the ship model.

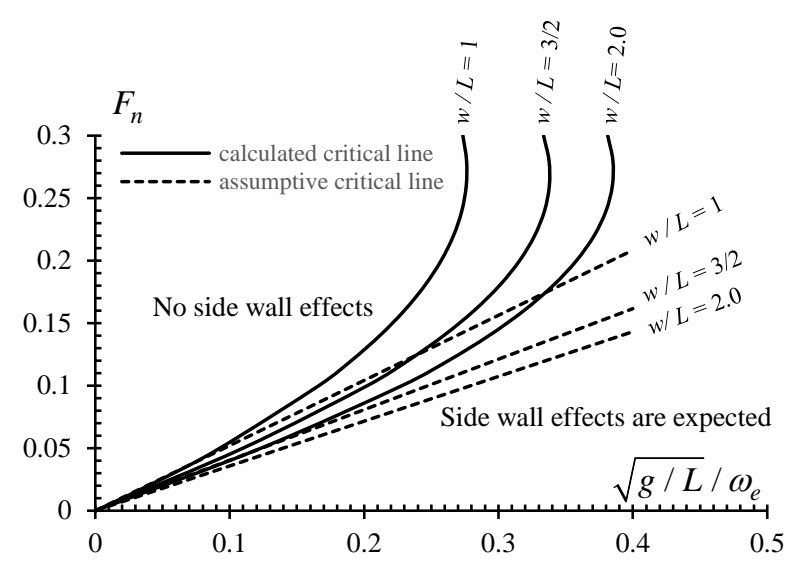

Fig. 20 Theoretical and numerical estimation of the critical lines showing whether the side wall effects are expected.

\section{Conclusions}

In this paper, a boundary element program based on the 3-D Rankine source method was developed to predict the side wall effects during ship model tests in the towing tank. We investigated the side wall effects on ship model tests both in calm water and waves. It was found that in calm water, the side wall effects can be neglected at $w / L>0.8$ in deep water. Significant side wall effects were observed at $w /$ $L=0.4$ due to the reflected waves from the side walls. The side wall effects were magnified at finite water depth. For a given ship model, the side wall effects in calm water testing are mainly determined by three factors: the width of the tank $(w)$, the water depth $(h)$ and the speed $(u)$ of the ship model. The side wall effects for a combination of $w$ and $u$ are uncertain. It depends on the reflected waves. When 
the crest of the reflected waves strikes the model's stern, the resistance becomes smaller than that in open water. When the model is struck by a trough, the wave-making resistance increases. The side wall effect on experiments in waves are more complicated than that in calm water due to the complexity of the wave systems. Compared to the calm water case, there is one more critical factor which determines the side wall effects in waves. That is parameter $\tau$. As the parameter $\tau<0.25$, the hydrodynamic coefficients (radiation forces) fluctuate violently away from the open sea results. As the parameter $\tau$ increases, the hydrodynamic coefficients gradually approach the open sea results and the side wall effects tend to diminish. At parameter $\tau<0.25$, a very interesting phenomenon was observed: the reflected and radiated waves were trapped within the towing tank and the resulting side wall effects became significant.

Based on large scale computations, two diagrams to of side wall effects (one in calm water and the other one in waves), were depicted to show whether the side wall effect was less than the permissible error to be included in the measured values. From the diagram in calm water, it was found that the side wall effects were always greater than $50 \%$ at $h / D<5$. It indicates that at shallow water $(h / D)$, the side wall effects must be taken into account, no matter how wide the tank is. From the diagram in waves, an evident discrepancy was observed between the theoretical estimation and numerical calculation, especially at the low frequency range. The theoretical estimation based on asymptotic far-field wave patterns under-estimated the range of the side wall effects. In practice, due to the near-field local waves, the side wall exists in a wider range.

\section{Acknowledgement}

This study was supported financially by the national natural science foundation of China (Grant no.51379095, 11372305) and the national basic research program of China (973 Program; Grant no.2013CB36100). We also thank Mr Thomas Sammut for language editing and proofreading this work.

\section{References}

Beck, R.F., Newman, J.N., Tuck, E.O., 1975. Hydrodynamic forces on ships in dredged channels. Journal of Ship Research 19 (3), 166-171.

Becker, E., 1958. Das Wellenbild einer unter der Oberfläche eines Stromes schwerer Flüssigkeit pulsierenden Quelle. Journal of Applied Mathematics and Mechanics 38 (9-10), 391-399.

Bunnik, T., 1999. Seakeeping calculations for ships, taking into account the non-linear steady waves, $\mathrm{PhD}$ thesis. Delft University of Technology, The Netherlands.

Chen, X.-N., Sharma, S.D., 2006. A slender ship moving at a near-critical speed in a shallow channel. Journal of Fluid Mechanics 291 (-1), 263.

Chen, X., Zhu, R., Ma, C., Fan, J., 2016. Computations of linear and nonlinear ship waves by higherorder boundary element method. Ocean Engineering 114, 142-153.

Doctors, L.L., 2015. Hydrodynamics of high-performance marine vessels. CreateSpace, Charleston, South Carolina.

Hess, J.L., Smith, A.M.O., 1964. Calculation of nonlifting potential flow about arbitrary threedimensional bodies. Journal of Ship Research 8 (2), 22-44.

Hosoda, R., 1976. Side wall effects of towing tank on the results of experiments in wave. Journal of the Society of Naval Architects of Japan 143, 52-60.

Huang, F., Yang, C., Noblesse, F., 2013. Numerical implementation and validation of the NeumannMichell theory of ship waves. European Journal of Mechanics - B/Fluids 42, 47-68.

Iwashita, H., 2001. Side wall effect of towing tank on measured unsteady waves in low frequency range, 16th International Workshop on Water Waves and Floating Bodies, Hiroshima, Japan.

Kajitani, H., 1983. The summary of the cooperative experiment on wigley parabolic model in Japan. Tokyo University. 
Kashiwagi, M., Ohkusu, M., 1989. Side-Wall Effects on Hydrodynamic Forces Acting on a Ship with Forward and Oscillatory Motions, 5th International Conference on Numerical Ship Hydrodynamics, Hiroshima, Japan.

Kashiwagi, M., Ohkusu, M., 1991. A new theory for side-wall interference effects on forward-speed radiation and diffraction forces. Ship Technology Research 38 (1), 17-48.

Kirsch, M., 1966. Shallow water and channel effects on wave resistance. Journal of Ship Reaearch 10 (3), 164-181.

Li, Y., Sclavounos, P.D., 2002. Three-dimensional nonlinear solitary waves in shallow water generated by an advancing disturbance. Journal of Fluid Mechanics 470 .

Maruo, H., 1966. A note on the higher order theory of thin ships. Bulletin of the Faculty of Engineering $15,1-21$.

Mei, C.C., Choi, H.S., 1987. Forces on a slender ship advancing near the critical speed in a wide canal. Journal of Fluid Mechanics 179, 59-76.

Newman, J.N., 1976. Linearized wave resistance, International Seminar on Wave resistance, Tokyo.

Newman, J.N., 1978. The theory of ship motion. Advances in Applied Mechanics 18 (221-283).

Newman, J.N., Poole, F.A.P., 1962. The wave resistance of a moving pressure distribution in a canal. Schiffstechnik 9, 21-26.

Noblesse, F., Hendrix, D., 1992. On the theory of potential flow about a ship advancing in waves. Journal of Ship Research 36 (1), 17-30.

Norrbin, N.H., 1974. Bank effects on a ship moving through a short dredged channel, Symposium on Naval Hydrodynamics (10th), Cambridge, Mass, June 24-28, 1974.

Prins, H.J., 1995. Time domain calculations of drift forces and moments, PhD Thesis. Delft University of Technology, The Netherlands.

Raven, H.C., 1994. Nonlinear ship wave calculations using the RAPID method, Sixth International Conference on Numerical Ship Hydrodynamics, Iowa City, Iowa, USA, pp. 95-118.

Takaki, M., 1979. Effects of breadth and depth of restricted waters on longitudinal motions in waves. Journal of the Society of Naval Architects of Japan 146, 173-184

Tarafder, S., 2007. Third order contribution to the wave-making resistance of a ship at finite depth of water. Ocean Engineering 34 (1), 32-44.

Tuck, E.O., 1978. Hydrodynamic Problems of Ships in Restricted Waters. Annual Review of Fluid Mechanics 10 (1), 33-46.

Yuan, Z.-M., Incecik, A., 2016. Investigation of ship-bank, ship-bottom and ship-ship interactions by using potential flow method, 4th MASHCON, Hamburg, Germany.

Yuan, Z.-M., Incecik, A., Dai, S., Alexander, D., Ji, C.-Y., Zhang, X., 2015a. Hydrodynamic interactions between two ships travelling or stationary in shallow waters. Ocean Engineering 108, 620-635.

Yuan, Z.-M., Incecik, A., Day, A., Jia, L., 2015b. Double Doppler shift theory on water waves generated by a translating and oscillating source, 30th Intl Workshop on Water Waves and Floating Bodies, Bristol, UK.

Yuan, Z.-M., Incecik, A., Day, A.H., 2014a. Verification of a new radiation condition for two ships advancing in waves. Applied Ocean Research 48, 186-201.

Yuan, Z.-M., Incecik, A., Jia, L., 2014b. A new radiation condition for ships travelling with very low forward speed. Ocean Engineering 88, 298-309.

Yuan, Z.-M., Ji, C.-Y., Incecik, A., Zhao, W., Day, A., 2016. Theoretical and numerical estimation of ship-to-ship hydrodynamic interaction effects. Ocean Engineering 121, 239-253. 
Yuan, Z.M., He, S., Paula, K., Incecik, A., Turan, O., Boulougouris, E., 2015c. Ship-to-Ship Interaction during Overtaking Operation in Shallow Water. Journal of Ship Research 59 (3), 172-187.

Zhao, W., Wolgamot, H.A., Taylor, P.H., Eatock Taylor, R., 2017. Gap resonance and higher harmonics driven by focused transient wave groups. Journal of Fluid Mechanics 812, 905-939. 